\title{
Bistatic Radar Detection of the Melting Layer ${ }^{1}$
}

\author{
RICHARD J. DOVIAK \\ National Severe Storms Laboratory, NOAA, Norman, Okla. 73060 \\ and Claude M. Weil \\ Environmental Protection Agency, Twinbrook Research Laboratory, National Environmental Research Center, \\ Research Triangle Park, N. C. 27711 \\ (Manuscript received 24 January 1972, in revised form 9 March 1972)
}

\begin{abstract}
Recent operation of the Valley Forge-Wallops Island bistatic radar link during two days characterized by light rain and drizzle has provided some detailed, bistatically derived information on atmospheric structure in the vicinity of Wallops Island. Data are presented that give unambiguous evidence of the simultaneous bistatic and monostatic radar detection of the melting layer. The height of the melting layer, as measured in the bistatic mode, has been confirmed by both radar backscatter measurements and radiosonde data. Methods used in interpreting forward-scatter data are discussed and approximate values for the layer thickness and reflectivity are derived.
\end{abstract}

\section{Introduction}

Layers of clear air scattering, observed with the Valley Forge-Wallops Island (VF-WI) bistatic radar system, are described by Doviak el al. (1971). This article describes results obtained under cloudy conditions with some trace of rain and compares simultaneous bistatic and monostatic radar observations as well as radiosonde data. Crane (1970) has made comparison of $\mathrm{cw}(3.88-\mathrm{cm}$ wavelength) transmission loss (along a $145-\mathrm{km}$ forward-scatter path) with backscatter radar data $(23 \mathrm{~cm}$ ), and Fernald and Dennis (1964) have compared monostatic and bistatic reflectivities $(5.1 \mathrm{~cm})$ when a fixed volume, common to the antenna main beams, encompassed a melting layer. Our experiment differs from Fernald's and Dennis' in that we use a scanning bistatic pulse volume (Rogers and Eccles, 1971) of sufficient resolution so that bistatically resolved "bright band" features are evident in photographic displays. Miller (1971) has shown that time discrimination techniques (i.e., use of bistatic pulse volumes) may be required in order to unambiguously associate bistatic reflectivity with targets contained in main beam common volumes.

The VF-WI bistatic radar possesses certain unique features; it is able to probe the atmosphere with narrow $\left(0.5^{\circ}\right)$ transmitter and receiver beamwidths and it contains a time discrimination capability as small as $0.1 \mu \mathrm{sec}$ with both stations synchronized in time to a stability of 1 part in $10^{11}$ per day. The link operates at a

\footnotetext{
IThis work was performed while the authors were at the Moore School of Electrical Engineering's Valley Forge Research and Education Center, Malvern, Pa.
}

frequency of $2.83 \mathrm{GHz}$ and the two radar sites are $248 \mathrm{~km}$ apart.

\section{Signature display of layered and diffuse targets}

The scattered signal received at Wallops Island from Valley Forge is usually recorded on an intensitymodulated polar display, with the radial coordinate representing time delay $\tau$, and the angular coordinate representing the receiver angle $\theta_{r}$. Fig. 1 gives an example of such a recording made at 1500 GMT 10 August 1970, a day characterized by light rain and drizzle. Local climatological data at Wallops Island show that a trace of rain was recorded during the hour

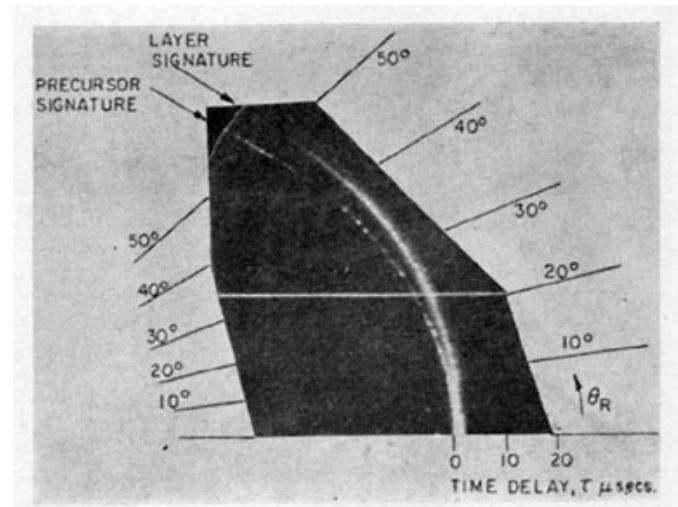

Fig. 1. Intensity-modulated polar display of signal received via forward scatter at 1500 GMT 10 August 1970 . The radial scale is calibrated in microseconds of time delay relative to the earliest time of arrival of signals; the angular scale is in degrees of elevation of the receiving antenna which scans the great circle plane illuminated by the transmitter beam fixed in elevation at $\theta_{1}=0.7^{\circ}$. 
that the bistatic data were being acquired and no measurable rainfall was recorded at several National Weather Service stations along the link (NOAA, 1970). The relevant operating parameters of the bistatic radar system were as follows: pulse width, $0.2 \mu \mathrm{sec}$; peak power of pulse, $840 \mathrm{~kW}$; minimum discernible signal sensitivity of receiver, $-115 \mathrm{dbm}$ (with film integration); and receiver bandwidth, $8 \mathrm{MHz}$.

Although a single record is presented, the features evident in Fig. 1 were repeatable for many scans and, furthermore, variation of system parameters (e.g., pulse width, bandwidth) produced changes in the polar display in accord with expectations. The interpretation of similar forward-scatter recordings, obtained on days of clear air, is presented by Doviak (1972).

The thin trace originating at $\tau=0$ (termed the "precursor") represents the earliest arriving signals, presumably received from low-altitude scatterers through the lower side lobes of the receiving antenna. The wider trace to the right of the precursor is assumed to be signals received through the receiver field of view from both diffusely distributed and layered scatterers located at higher altitudes. The bright band (region of enhanced intensity) within this trace represents scattering from a well-defined layer and is termed a "layer signature." The altitude of the layer which creates this signature is computed by measuring the time delay of the signature for various receiver elevation angles. The broader and less distinct portion of the wide trace is assumed to represent returns from diffusely distributed scatterers that are located above and below the layer. The horizontal trace evident in Fig. 1 is a calibration mark used for determining the angular scale. Although an additional layer-type signature appears for angles $>40^{\circ}$, we have not established whether this is evidence of an elevated layer or side-lobe coupling through the melting layer.

\section{Determination of target locations}

Fig. 2 gives a presentation of atmospheric targets located with reference to height above and distance from the receiver station at Wallops Island. The elliptical curves represent loci of constant time delay relative to the great circle path propagation time. Using these, we can determine the altitude, approximate thickness, and horizontal extent of the layer whose signature appears in Fig. 1. The time delay data derived from Fig. 1 give the results shown in Fig. 2 ; i.e., a layer centered at an altitude of $3.8 \mathrm{~km}$, approximately $500 \mathrm{~m}$ thick, and having a horizontal extent of more than 35 $\mathrm{km}$ (beyond this point, the layer signature of Fig. 1 is no longer resolvable). The approximate boundaries of the diffusely distributed targets which have been detected during forward-scatter operations are indicated in Fig. 2 by dashed lines. The uncertainty of target locations in Fig. 2 is determined by estimated errors of $\pm 0.2^{\circ}$ in elevation angle and $\pm 0.2 \mu \mathrm{sec}$ in time delay.

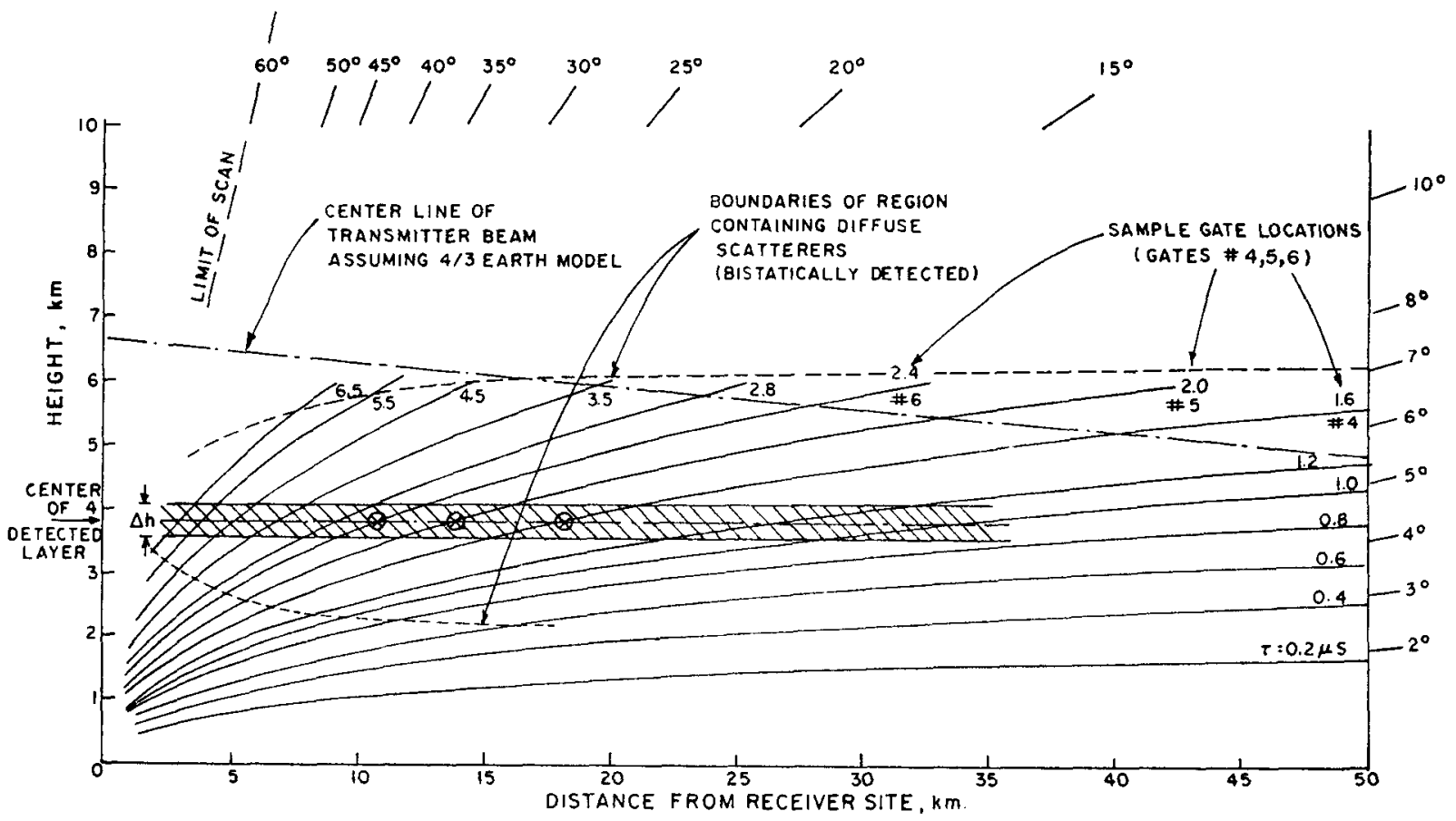

FIG. 2. Distance-height locations of atmospheric targets showing regions of bistatically resolved layered and diffusely distributed targets. Elliptical time delay curves are marked in units of microseconds of time delay referenced to great circle path propagation time. Gate locations No. 4-6 are regions (of constant time delay) whose scatterintensity vs $\theta_{r}$ is displayed in Fig. 3. 


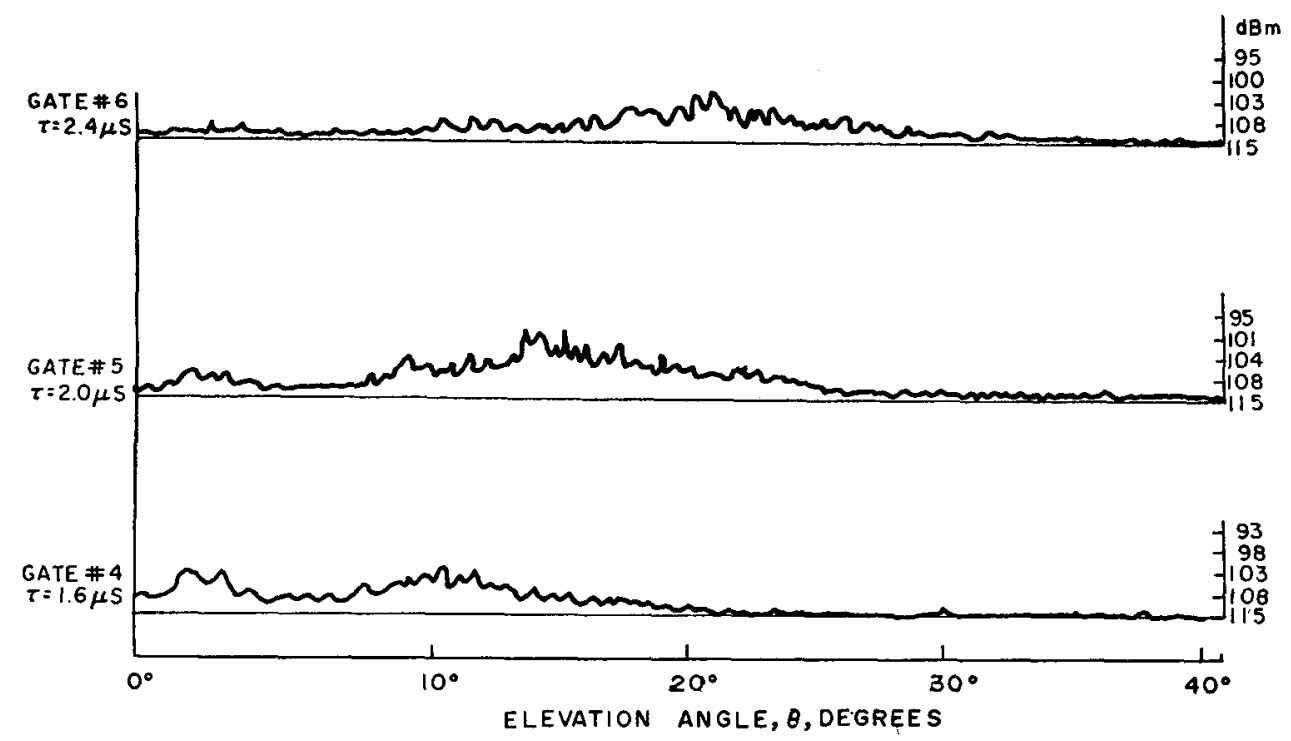

FIg. 3. Amplitude versus receiver elevation angle for the three sampled spheroidal shells indicated in Fig. 2. Gate width $=0.1 \mu$ sec, gate delays $t_{d}=1.6,2.0,2.4 \mu \mathrm{sec}$ for gate positions $4,5,6$, respectively. Recordings were made at same time that data of Fig. 1 were photographed.

The location of the transmitter beam center line, assuming a $\frac{4}{3}$-earth radius propagation model, is also shown in Fig. 2. Note that this location lies above most of the region from which forward-scatter returns were obtained (with the transmitter elevation angle fixed at $0.7^{\circ}$ ). Thus, the layer of enhanced reflectivity displayed in Fig. 1 may be illuminated by the lower gain portions of the transmitter beam.

An approximate value for the layer thickness is obtainable by measuring the width of the layer signature $(\mu \mathrm{sec})$ along a line of constant angle and subtracting one pulse width from this. For example, at an elevation angle of $30^{\circ}$ the layer signature has an approximate width of $0.7 \mu \mathrm{sec}$. Subtracting the value of the pulse width $(0.2 \mu \mathrm{sec})$ and using the time delay curves of Fig. 2 gives an approximate layer thickness of $500 \mathrm{~m}$. This computation was then repeated for additional elevation angles and approximately the same value of layer thickness was obtained in each case.

\section{Identification of melting layer}

Temperature-altitude profiles were obtained from radiosonde observations at Wallops Island and Washington, D. C., about $3 \frac{1}{2}$ hours before the forwardscatter measurements were conducted. These profiles show a $0 \mathrm{C}$ level at an altitude of $3.8 \mathrm{~km}$, a height that correlates well with the layer indicated in Fig. 2. Hence, it is reasonable to assume that the bright band of Fig. 1 is the bistatic representation of the melting layer. In addition, the Wallops Island sounding showed a $0 \mathrm{C}$ isothermal layer of $500 \mathrm{~m}$ thickness which is excellent evidence of melting layer effects.

Similar data were also obtained on 23 July 1970. For this date, both monostatic and bistatic radar data showed the melting layer to be centered at an altitude of $4.3 \mathrm{~km}$.

\section{Amplitude measurements}

Amplitude measurements were made of the received signals scattered from the melting layer region. These signals, arriving at Wallops Island, were sampled with gates spaced at fixed time delays of 1.6, 2.0 and $2.4 \mu \mathrm{sec}$ relative to the zero time reference $(\tau=0)$ and having approximately $0.1 \mu \mathrm{sec}$ width. These amplitude data were recorded as the receiving antenna scanned (at a rate of $1^{\circ} \mathrm{sec}^{-1}$ ) the region illuminated by the fixed transmitting antenna. Referring to Fig. 2, it is evident that as the receiver antenna is scanning in the great circle plane, each gate samples a portion of the spheroidal shell that is centered at the appropriate time delay curve, has a thickness of approximately one pulse width $\Delta \tau$, and is bounded along the shell by the beamwidth of the receiving antenna. Consequently, as $\theta_{r}$ increases from small angles, we are sampling a region in space which passes through the layer from above and ends below the layer. Referring to Fig. 3 , which shows gated amplitude data for gate positions 4,5 and 6 , there is a well-defined peak of signal intensity at approximately $\theta_{r}=11^{\circ}, 15^{\circ}$ and $20^{\circ}$, respectively. The circled crosses in Fig. 2 indicate intersections of receiver beam axis (at each of these angles) and time delay curves of each respective gate. The coincidence of the maximum echo strength with the center of the layer location obtained from Fig. 1 further confirms the value of $3.8 \mathrm{~km}$ deduced for the melting layer height.

The purpose of the following paragraphs is to confirm that the bistatically derived reflectivity is consistent with that expected for the meteorological conditions 
existing at the time of the observations. Furthermore, a simplified equation for the bistatic pulse volume is derived. This equation appears to be a useful approximation, for the stipulated conditions, and avoids the lengthy solution involving the convolution of the two radiation patterns with the reflectivity pattern (Rogers and Eccles, op. cit.).

The received power obtained in bistatic operations is a function of both the time delay $t_{d}$ (referenced to straight line propagation between transmitter and receiver) and the receiver elevation angle $\theta_{r}$, and is given by (Skolnik, 1962)

$$
P_{r}\left(l_{d}, \theta_{r}\right)=\frac{P_{t} G_{t} G_{r} \lambda^{2}}{(4 \pi)^{3} R_{t}^{2} R_{r}^{2}} \sigma\left(t_{d}, \theta_{r}\right) \cos ^{2}\left(\theta_{r}+\theta_{t}\right)
$$

where $P_{t}$ is the peak transmitter power, $G_{t}$ and $G_{r}$ are the antenna gains for the transmitting and receiving antennas, respectively $\left(G_{r}=1.2 \times 10^{5}\right), \quad R_{t}$ and $R_{r}$ represent distances to the sample volume from the transmitter and receiver, respectively, $\lambda$ is the wavelength, and $\sigma$ the cross section for the sample volume. We assume here that the particle size is sufficiently small (Rayleigh approximation) so that the particulate bistatic cross section differs, for the vertical polarization used in these experiments, from the monostatic cross section by the cosine squared term in (1). Thus, $\sigma$ is given approximately by

$$
\sigma=\frac{\pi^{5} Z_{e} V_{s}}{\lambda^{4}}
$$

where $Z_{e}$ is the average effective reflectivity factor of the scatterers within the sample volume $V_{s}$. Note that both $Z_{e}$ and $V_{s}$ are functions of $t_{d}$ and $\theta_{r}$. The sample volume $V_{s}$ is assumed to be given by the product

$$
V_{s}=A\left(\theta_{r}, t_{d}\right) \cdot L\left(\theta_{r}, l_{d}, \Delta \tau\right),
$$

where $A$ is the area circumscribed by the half-power receiver beamwidth intersecting with a plane that is tangential to the spheroidal surface, and $L$ is the length, proportional to the pulse width $\Delta \tau$, of a line segment that is perpendicular to the spheroidal surface.

Assuming that $\theta_{r}$ is limited to angles $<45^{\circ}$ and that the time delays being considered are such that $t_{d} \ll D / c$ ( $D$ is the distance separating transmitter and receiver, $c=3 \times 10^{8} \mathrm{~m} \mathrm{sec}^{-1}$ ), then it is easy to show that $V_{s}$ is given by

$$
V_{s}=\pi\left(\frac{t_{d}}{\Delta \tau}\right)^{2} \frac{\Omega_{r}^{2} L^{3}}{\sin ^{2} \theta_{r} \sin \left(\theta_{r}-\alpha\right)}
$$

where $\Omega_{r}$, the half-power beamwidth of the receiving antenna $\left(8.5 \times 10^{-3} \mathrm{rad}\right)$ is assumed to be less than $\theta_{r}$; $\alpha$ is the angle that the tangent plane makes with respect to the straight line passing through transmitter and receiver, i.e.,

$$
\alpha=\tan ^{-1}\left(\frac{1}{2} \tan \theta_{r}-\frac{c t_{d}}{D \tan \theta_{r}}\right)
$$

$G_{t}$ is assumed constant over $V_{s}$; and $L$ is given by

$$
L=\frac{c \Delta \tau \tan \theta_{r}}{\left(\frac{2 c t_{d}}{D}+\tan ^{2} \theta_{r}\right)} .
$$

Using Eqs. (1)-(6), we now derive a value of $Z_{e}$ for the melting layer, using a sample volume within that layer defined by $l_{d}=2.4 \mu \mathrm{sec}, \Delta \tau=0.2 \mu \mathrm{sec}$, and $\theta_{r}=21^{\circ}$. Substituting for the remaining parameters gives the expression

$$
Z_{e} G_{t}=2 \times 10^{18} P_{r}
$$

where $Z_{e}$ is in units of $\mathrm{mm}^{6} \mathrm{~m}^{-3}$, and $P_{r}$ is in watts. From Fig. 3, we note that for gate position 6, $P_{r}=-103$ $\mathrm{dbm}$ at $\theta_{r}=21^{\circ}$. The transmitting antenna gain $G_{i}$ for this location of $V_{s}$ is obtainable from recorded plots made of the transmitter antenna radiation pattern. This is estimated to be approximately $30 \mathrm{db}$ assuming the main beam axis to be positioned as indicated in Fig. 2. Substituting both of these values into (6) gives a reflectivity of $Z_{e}=100 \mathrm{~mm}^{6} \mathrm{~m}^{-3}$. This is well within the range of values normally expected with the very light precipitation observed by the radar operators at the time and corroborated by the official rainfall record at cooperative National Weather Service Stations.

\section{Conclusions}

The bistatic resolution of atmospheric target locations using a sample volume of space defined by the receiver field of view and a spheroidal shell of constant propagation delay and thickness related to the transmitted pulse width has provided an interpretation of forward-scatter multipath signal structure that gives unambiguous evidence of the existence of a layer of scatterers having a reflectivity larger than the region immediately above and below the layer. This layer of enhanced bistatic reflectivity is consistent in height with the location of the monostatically detected melting layer and also with the melting level deduced from radiosondes released in the vicinity of the bistatic link.

Since simultaneous bistatic and monostatic radar cross-section measurements can yield information on the structure of the irregularly shaped melting snowflake, it appears that a bistatic system, having pulse volume resolution, would be a useful supplement to a monostatic radar. The simultaneous use of monostatic and bistatic radars to deduce rainfall rate has been suggested by Eccles and Rogers (1968). The results of the work reported herein should provide a firmer experimental base on which future experiments could be designed. 
Acknowledgments. The authors wish to express their appreciation to Isadore Katz and Thomas Konrad of the Applied Physics Laboratory, The Johns Hopkins University, for providing them with the facility and manpower at Wallops Island. We also wish to thank Jack Howard and John Shinn, supervisors of the Wallops Island and Valley Forge radar sites, respectively, for their cooperation. Finally, we acknowledge the suggestions of Drs. E. Kessler of NSSL and K. Hardy of AFCRL who strengthened many aspects of this work.

The Valley Forge radar site was supported by the National Science Foundation under Grant GA-1704, and the Moore School of Electrical Engineering, University of Pennsylvania.

\section{REFERENCES}

Crane, R. K. 1970: A comparison between monostatic and bistatic scattering from rain and thin turbulent layers. Tech. Note 1970-29, Lincoln Laboratory, M.I.T., Lexington, Mass.
Doviak, R. J., 1972: Comparison of bistatic and monostatic radar detection of clear air atmospheric targets. Proc. AIAA 10th Aerospace Science Meeting, San Diego, Calif., 72-175.

_- J. Goldhirsh and A. R. Miller, 1971: Time resolution of tropospheric layers with the Valley Forge-Wallops Island forward scatter probe. IEEE Trans. Antennas Propagation, AP-19, 714-716.

Eccles, P., and P. Rogers, 1968: Relationship between rainfall rate and other measurable parameters of precipitation, the bistatic radar equation. Preprints of Papers 13th Radar Meteorology Conf., Montreal, Amer. Meteor. Soc., 364-369.

Fernald, F. G., and A. S. Dennis, 1964: Measurement of forwardscatter cross sections in the melting layer. Preprints of Papers, 1964 World Conf. Radio Meteorology, Boulder, Colo., Amer. Meteor. Soc., 178-181.

Miller, A. R., 1971: Resolvability of main beam and side lobe scattering volumes in a bistatically probed atmosphere. Ph.D. dissertation, University of Pennsylvania, Philadelphia.

NOAA, Environmental Data Service, 1970: Hourly precipitation data-Maryland and Delaware. Vol. 20, No. 8, Asheville, N. C.

Rogers, P. J., and P. Eccles, 1971 : The bistatic radar equation for randomly distributed targets. Proc. IEEE, 59, 1019-1021.

Skolnik, Merril I., 1962 : Introduction to Radar Systems. New York, McGraw-Hill, 648 pp. 